\title{
ESTUDO ESTATÍSTICO DA PRODUÇÃO DE CARVÃO ATIVADO A PARTIR DAS FIBRAS DE SISAL
}

\author{
C. F. S. ROMBALDO ${ }^{1,2}$, A. C. L. LISBOA ${ }^{1}$, M. O. A. MENDEZ ${ }^{2}$ e A. R. COUITNHO ${ }^{2}$ \\ ${ }^{1}$ Universidade Estadual de Campinas, Faculdade de Engenharia Química \\ ${ }^{2}$ Universidade Metodista de Piracicaba, Laboratório de Materiais Carbonosos \\ E-mail para contato: cfrombaldo@ unimep.br
}

\begin{abstract}
RESUMO - O presente trabalho teve como objetivo investigar a produção de carvão ativado a partir das fibras de sisal, que consiste de uma matéria prima renovável, de enorme disponibilidade e de procedência nacional. Inicialmente as fibras de sisal in natura foram submetidas à carbonização em atmosfera inerte, seguido de ativação física em atmosfera oxidante, com dióxido de carbono. Foram realizados ensaios em diferentes fluxos de gás ativante, assim como diferentes tempos de residência e temperaturas de ativação. Como respostas do planejamento estatístico foram obtidas: o rendimento em massa do processo, a massa específica real e a área superficial específica dos carvões ativados produzidos. Os resultados indicaram que o tempo de residência é uma das variáveis estatisticamente mais significativas para o processo de produção de carvão ativado a partir das fibras de sisal, apresentando efeito negativo para a resposta rendimento e efeito positivo para a área superficial específica. $\mathrm{O}$ rendimento médio do processo foi de $23 \%$. O maior valor de área superficial específica, de $580 \mathrm{~m}^{2} \cdot \mathrm{g}^{-1}$, foi alcançado no nível superior das variáveis estudadas.
\end{abstract}

\section{INTRODUÇÃO}

O sisal (Agave sisalana) é uma planta originária do continente Norte Americano e das Ilhas Caribenhas. Suas propriedades são conhecidas desde as épocas mais remotas pelos primitivos habitantes do México. Dada a sua facilidade de aclimatação, a Agave foi levada do México para outras partes do mundo, passando a ser comercialmente cultivada (Oashi, 1999). Além dos usos comuns do sisal, como fios biodegradáveis utilizados para artesanatos e sacarias, adubo orgânico, ração animal e biofertilizantes, tem crescido o número de pesquisas que procuram viabilizar a utilização das fibras do sisal na indústria, utilizando-as como, por exemplo, para substituir a fibra de vidro na indústria automobilística (Ramesh et al., 2013), para conferir maior resistência, na indústria da construção civil (Arruda Filho et al., 2012; Farias Filho et al., 2010), para atuar como reforço na produção de compósitos poliméricos (Ku et al., 2011; Li et al., 2000), e para a produção de carvão ativado (Chen and Zeng, 2003; Mestre et al., 2011) dentre outras aplicações.

Hoje, no Brasil o sisal é o principal produto agroindustrial do semiárido brasileiro. A cadeia do sisal gera empregos diretos e indiretos de 700 mil pessoas. Estima-se a existência de até 35 mil produtores. O Brasil é o maior produtor e exportador mundial da fibra e, no ano de 2009, cerca de 50\% da produção mundial do sisal foi brasileira (Aquino, 2011). Em 2011 a produção 
brasileira foi de 111 mil t. A Bahia produziu 95,8\% deste total, com participação menor de outros estados, como: Paraíba (3,5\%), Ceará $(0,4 \%)$ e do Rio Grande do Norte $(0,3 \%)$.

O objetivo do presente trabalho foi investigar por meio de um planejamento fatorial completo (Barros Neto et al., 2003) a produção de carvão ativado a partir das fibras de sisal, que é uma matéria prima renovável, de procedência nacional e de baixo custo.

\section{MATERIAIS E MÉTODOS}

Foram produzidas amostras de CA a partir da ativação física das fibras de sisal em atmosfera de $\mathrm{CO}_{2}$. Foi utilizado o sistema apresentado na Figura 1, que consiste de: um forno elétrico tubular vertical e estático (1), um reator de quartzo (2) e um sistema de fluxo de $\mathrm{N}_{2}$ (7) e $\mathrm{CO}_{2}$ (8). A temperatura e a taxa de aquecimento foram monitoradas e controladas por um controlador de potência (3), por meio de medidas das temperaturas interna e externa com termopares (4) e (5), respectivamente. O conjunto experimental foi colocado sob um sistema de exaustão de gases (6). Em cada ensaio foram utilizadas $40 \mathrm{~g}$ de fibras de sisal, com $300 \mathrm{~mm}$ comprimento.

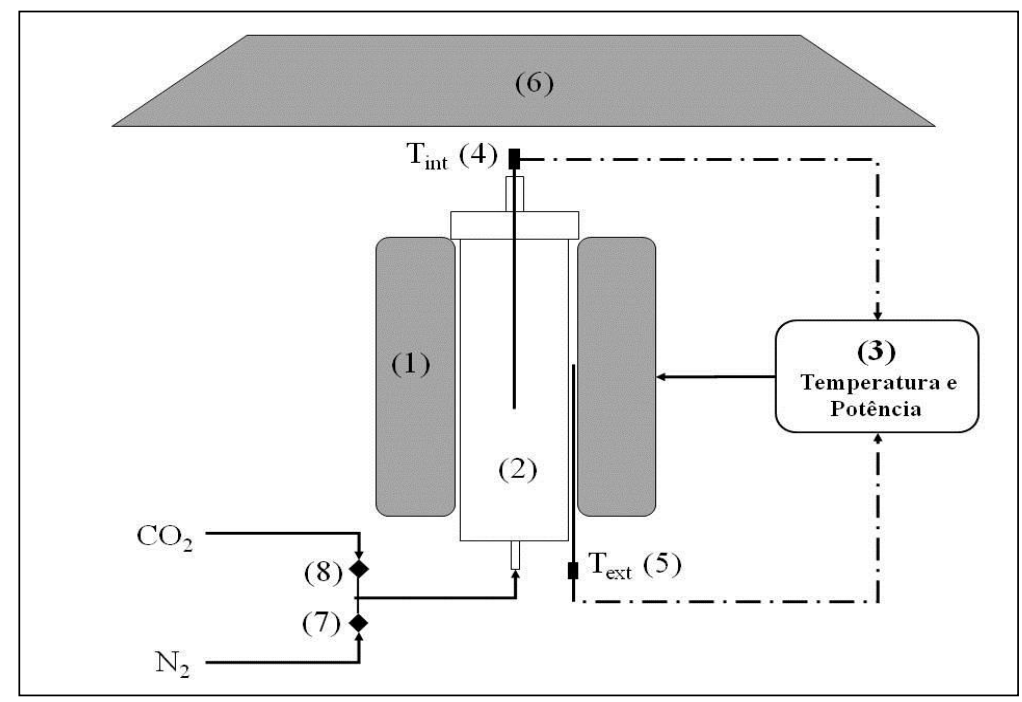

Figura 1 - Sistema estático utilizado na carbonização e ativação das FSAC.

A influência das variáveis de processo de ativação foi investigada por meio de um planejamento estatístico fatorial completo do tipo $2^{3}$, com três fatores, em dois níveis de intensidade $(-1,+1)$ com triplicata no ponto central, obtendo como resposta do planejamento o rendimento em massa do carvão ativado, massa específica real e a área superficial específica (ASE) das fibras ativadas com $\mathrm{CO}_{2}$ (FSAC). A Tabela 1 apresenta as variáveis estudadas e seus respectivos níveis escolhidos para realização dos ensaios. Todos os experimentos foram realizados em ordem aleatória para minimizar ao máximo os erros sistemáticos do processo

O rendimento em massa foi obtido com base na massa de fibra de sisal in natura utilizada 
no processo. A determinação da massa específica real foi feito por deslocamento do gás Hélio em equipamento da Quantachrome ${ }^{\circledR}$, modelo Ultrapycnometer 1000. A ASE foi determinada por adsorção de nitrogênio a $77 \mathrm{~K}$ em equipamento da Quantachrome ${ }^{\circledR}$, modelo Autosorb-1, através do modelo BET.

Tabela 1 - Níveis e variáveis estudadas no processo de ativação com $\mathrm{CO}_{2}$ das fibras de sisal

\begin{tabular}{c|c|c|c}
\hline \multirow{2}{*}{ Variáveis } & \multicolumn{3}{|c}{ Níveis } \\
\cline { 2 - 4 } & $\mathbf{- 1}$ & $\mathbf{0}$ & $\mathbf{+ 1}$ \\
\hline$\left(\mathrm{X}_{1}\right)$ Temperatura da isoterma de ativação $\left({ }^{\circ} \mathrm{C}\right)$ & 700 & 750 & 800 \\
\hline$\left(\mathrm{X}_{2}\right)$ Tempo de residência da ativação $(\mathrm{min})$ & 30 & 75 & 120 \\
\hline$\left(\mathrm{X}_{3}\right)$ Fluxo de $\mathrm{CO}_{2}\left(\mathrm{~mL} \cdot \mathrm{min}^{-1}\right)$ & 100 & 150 & 200 \\
\hline
\end{tabular}

\section{RESULTADOS E DISCUSSÕES}

A Tabela 2 mostra a matriz do planejamento experimental e as respostas: rendimento $(\eta)$, massa específica real $(\rho)$ e área superficial específica (ASE) das FSAC.

Tabela 2 - Matriz do planejamento experimental com as respostas: $\eta, \rho$ e ASE das FSAC

\begin{tabular}{c|c|c|c|c|c|c}
\hline Ensaio & $\mathbf{X}_{\mathbf{1}}$ & $\mathbf{X}_{\mathbf{2}}$ & $\mathbf{X}_{\mathbf{3}}$ & $\begin{array}{c}\eta \\
(\boldsymbol{\%})\end{array}$ & $\begin{array}{c}\rho \\
\left(\mathbf{g . c m}^{-\mathbf{3}}\right)\end{array}$ & $\begin{array}{c}\mathbf{A S E} \\
\left(\mathbf{m}^{\mathbf{2}} \mathbf{.} \mathbf{- 1}\right)\end{array}$ \\
\hline FSAC1 & -1 & -1 & -1 & 26,29 & 1,77 & 84,2 \\
\hline FSAC2 & 1 & -1 & -1 & 24,30 & 1,81 & 238,3 \\
\hline FSAC3 & -1 & 1 & -1 & 23,40 & 1,83 & 442,8 \\
\hline FSAC4 & 1 & 1 & -1 & 21,71 & 1,95 & 488,8 \\
\hline FSAC5 & -1 & -1 & 1 & 26,18 & 1,81 & 120,9 \\
\hline FSAC6 & 1 & -1 & 1 & 24,53 & 1,94 & 467,2 \\
\hline FSAC7 & -1 & 1 & 1 & 23,91 & 1,92 & 465,2 \\
\hline FSAC8 & 1 & 1 & 1 & 15,93 & 1,93 & 581,7 \\
\hline FSAC9 & 0 & 0 & 0 & 22,89 & 1,78 & 461,6 \\
\hline FSAC10 & 0 & 0 & 0 & 24,40 & 1,79 & 458,4 \\
\hline FSAC11 & 0 & 0 & 0 & 24,08 & 1,85 & 427,0 \\
\hline
\end{tabular}

Os dados da Tabela 2 indicam rendimento na faixa de $16 \%$ à $26 \%$ em carvão ativado. $\mathrm{O}$ menor rendimento, $16 \%$, foi alcançado nos níveis superiores das variáveis estudadas, pois quanto maior o tempo de residência e a maior a temperatura de ativação maior a quantidade de matéria prima consumida. O inverso é observado: em condições amenas de temperatura e tempo de ativação, obtêm-se os maiores rendimentos. 
A massa específica real das fibras de sisal in natura é de $1,58 \mathrm{~g} . \mathrm{cm}^{-3}$. Por outro lado, as amostras ativadas com $\mathrm{CO}_{2}$ apresentaram massa específica real entre $1,77 \mathrm{~g} . \mathrm{cm}^{-3}$ e $1,94 \mathrm{~g} \cdot \mathrm{cm}^{-3}, \mathrm{o}$ que indica uma mudança na estrutura porosa da fibra de sisal.

ASE apresentou valores entre $84 \mathrm{~m}^{2} \cdot \mathrm{g}^{-1}$ e $582 \mathrm{~m}^{2} \cdot \mathrm{g}^{-1}$, sendo a variação ocorrida em função das condições de processo. Destaca-se que o valor alcançado de $582 \mathrm{~m}^{2} \mathrm{~g}^{-1}$ é compatível com os carvões ativados produzidos a partir de outras fibras naturais, como juta (Phan et al., 2006; Asadullah et al., 2010), celulose (Mohamed et al., 2010).

A partir das repostas obtidas foi feito um estudo estatístico no sentido de verificar a significância de cada variável para o processo, cujos resultados estão apresentados na Tabela 3 e na Tabela 4, para as respostas rendimento e ASE, respectivamente. Todas as tabelas apresentam os valores dos efeitos principais, das interações de dois e de três fatores, assim como seus erros padrões e seus respectivos intervalos de confiança para cada resposta estudada.

Para a resposta massa específica real, o tratamento estatístico mostrou que para um nível de 95\% de confiança, todos os efeitos principais, assim como os efeitos de interações de dois e três fatores não são estaticamente significativos, pois seus intervalos de confiança compreenderam o zero.

Tabela 3 - Efeitos, erros padrões e intervalo de confiança para a resposta rendimento

\begin{tabular}{|c|c|c|}
\hline & & tervalo Confiança \\
\hline Média: & $23,42 \pm 0,24$ & {$[22,2924,45]$} \\
\hline \multicolumn{3}{|l|}{ Efeitos Principais } \\
\hline 1 (Temperatura) & $-3,33 \pm 0,56$ & {$\left[\begin{array}{ll}-5,75 & -0,91\end{array}\right]$} \\
\hline 2 (Tempo) & $-4,09 \pm 0,56$ & {$\left[\begin{array}{ll}-6,51 & -1,67]\end{array}\right.$} \\
\hline 3 (Fluxo) & $-1,29 \pm 0,56$ & {$\left[\begin{array}{ll}-3,71 & 1,13\end{array}\right]$} \\
\hline \multicolumn{3}{|c|}{ Interação de dois fatores } \\
\hline 12 & $-1,51 \pm 0,56$ & {$\left[\begin{array}{ll}-3,91 & 0,91]\end{array}\right.$} \\
\hline 13 & $-1,49 \pm 0,56$ & {$\left[\begin{array}{ll}-3,93 & 0,93\end{array}\right]$} \\
\hline 23 & $-1,35 \pm 0,56$ & {$\left[\begin{array}{ll}-3,77 & 1,07]\end{array}\right.$} \\
\hline \multicolumn{3}{|c|}{ Interação de três fatores } \\
\hline 123 & $-1,66 \pm 0,56$ & {$\left[\begin{array}{ll}-4,08 & 0,76\end{array}\right]$} \\
\hline
\end{tabular}


Tabela 4 - Efeitos, erros padrões e intervalos de confiança para a resposta ASE

\begin{tabular}{ccc}
\hline & & Intervalo Confiança \\
\hline Média & $385,10 \pm 5,76$ & {$\left[\begin{array}{ll}360,30 & 409,90\end{array}\right]$} \\
\hline Efeitos Principais & & \\
$\mathbf{1}$ (Temperatura) & $165,73 \pm 13,52$ & {$\left[\begin{array}{ll}107,55 & 223,89\end{array}\right]$} \\
$\mathbf{2}$ (Tempo) & $266,98 \pm 13,52$ & {$\left[\begin{array}{ll}208,81 & 325,14\end{array}\right]$} \\
$\mathbf{3}$ (Fluxo) & $95,23 \pm 13,52$ & {$\left[\begin{array}{ll}37,05 & 153,40\end{array}\right]$} \\
& & \\
Interação de dois fatores & & \\
$\mathbf{1 2}$ & $-84,47 \pm 13,52$ & {$\left[\begin{array}{ll}-142,65 & -26,31\end{array}\right]$} \\
$\mathbf{1 3}$ & $65,67 \pm 13,52$ & {$[7,50123,84]$} \\
$\mathbf{2 3}$ & $-37,57 \pm 13,52$ & {$\left[\begin{array}{lll}-95,74 & 20,59\end{array}\right]$} \\
& & \\
Interação de três fatores & & {$\left[\begin{array}{ll}-88,59 & 27,74\end{array}\right]$} \\
$\mathbf{1 2 3}$ & $-30,42 \pm 13,52$ & \\
\hline
\end{tabular}

O tratamento estatístico (Tabela 3), para a resposta rendimento mostra que para um intervalo de confiança de $95 \%$ os efeitos significativos variáveis temperatura e tempo de ativação, ambas com valor negativo. Indicando que quanto maior o tempo e a temperatura de ativação empregados durante o processo, menor será o rendimento em carvão ativado. Este comportamento comprova as variações observadas na Tabela 2.

A Tabela 4 mostra que para o mesmo nível de confiança todos os efeitos principais e as interações: temperatura e tempo de ativação (12), e temperatura e fluxo (13) são estatisticamente significativas, pois nos seus respectivos intervalos de confiança não está compreendido o zero.

Para a ASE, todos os efeitos principais e a interação temperatura e fluxo (13) são positivos, indicando que há uma proporcionalidade entre um aumento no nível das variáveis estudadas e a ASE, de forma que a combinação entre esses dois efeitos potencializa a formação e/ou alargamentos de poros e por consequência aumenta a ASE. O tempo de ativação foi a variável que apresentou o maior efeito dentre as variáveis estudadas, indicando maior capacidade de aumento na ASE.

A interação temperatura e tempo de ativação (12) apresentou efeito negativo sobre a ASE, ou seja, geram uma diminuição na ASE se aumentados simultaneamente. Isso ocorre em virtude do material ser exposto a temperaturas altas por longos períodos. A análise de variância para a resposta ASE está apresentada na Tabela 5. 
Para a resposta massa específica real, o tratamento estatístico mostrou que para um nível de 95\% de confiança, todos os efeitos principais, assim como os efeitos de interações de dois e três fatores não são estaticamente significativos, pois seus intervalos de confiança compreenderam o zero.

Tabela 5 - Análise de variância da resposta ASE das FSAC

\begin{tabular}{cccc}
\hline Fonte de Variação & $\begin{array}{c}\text { Soma } \\
\text { Quadrática }\end{array}$ & N de g. l. & $\begin{array}{c}\text { Média } \\
\text { Quadrática }\end{array}$ \\
\hline Regressão & 243190,039 & 7,000 & 34741,434 \\
Resíduos & 17574,361 & 3,000 & 5858,120 \\
Falta Ajuste & 16843,241 & 1,000 & 16843,241 \\
Erro Puro & 731,120 & 2,000 & 365,560 \\
\hline Total & 260764,400 & 10,000 & \\
\hline \% de variação explicada: 93,26 & & \\
\% máxima de variação explicável: 99,72 & & \\
\hline
\end{tabular}

Os os valores da Tabela 5 indicam que há evidência de falta de ajuste para um modelo linear da resposta ASE, apesar da variação percentual explicável ser alta, pois os dados mostram que um teste $\mathrm{F}$ para falta de ajuste é: $\mathrm{MQ}_{\mathrm{faj}} / \mathrm{MQ}_{\mathrm{ep}}=46,08$, por este valor ser maior que $\mathrm{o}$ $\mathrm{F}_{1,2}=18,51$. Esse fato também pode ser explicado pelas interações: temperatura e tempo (12) e temperatura e fluxo (13), terem se mostrado significativas para o planejamento estudado.

Pode-se notar que há evidência de uma curvatura significativa para a resposta área superficial específica, o que mais uma vez descarta a possibilidade de ajuste dos dados a um modelo linear:

$$
\begin{aligned}
& \text { Pontocentral }: \bar{y}_{c}=449,00 \\
& \text { Vértices }: \bar{y}_{v}=364,23 \\
& -\overline{y_{v}}-\bar{y}_{c}=-84,76
\end{aligned}
$$

Para comprovar que a diferença é significativa, fez-se a estimativa de seu erro:

$$
\begin{aligned}
& V\left(\bar{y}_{v}\right)=V\left(\bar{y}_{c}\right)=\frac{s^{2}}{3}=\frac{365,56}{3}=121,85 \\
& \text { ErroPadrão }=\sqrt{121,85}=11,038
\end{aligned}
$$

Multiplicando 11,038 por $\mathrm{t}_{2}=4,303$ ( 2 graus de liberdade e $95 \%$ de confiança), pode-se concluir que o valor limite para a significância da diferença é de 47,5, o que mais uma vez mostra 
evidência de uma curvatura na superfície de reposta. Como a resposta média no centro é superior à média das respostas nos vértices, à superfície deve ser convexa.

A Figura 2 apresenta a superfície de resposta para a variável ASE e o modelo obtido está apresentado na Equação 1, onde $T$ é a temperatura de ativação codifica e $t$ é o tempo de ativação codificado.

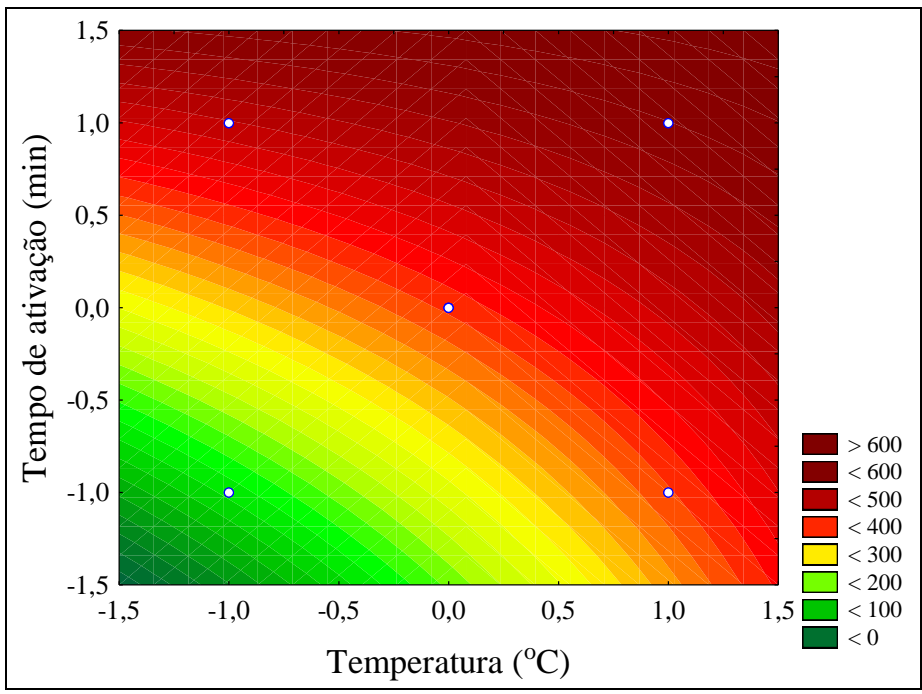

Figura 2 - Superfície de resposta para resposta ASE em função do tempo e ativação e da temperatura de ativação das FSAC.

$$
A S E=385,1+82,9 T+133,5 t-42,2 T t
$$

\section{CONCLUSÕES}

Os resultados obtidos indicam que o tempo de residência é a variável estatisticamente mais significativa no processo de obtenção de carvão ativado a partir da fibra de sisal. Para a resposta rendimento, o feito do tempo de residência se apresentou como negativo, portanto quanto maior o nível da variável menor o rendimento em CA. Para a reposta ASE o efeito do tempo de residência se mostrou positivo, indicando uma proporcionalidade entre o tempo e ASE do carvão ativado obtido.

Para a resposta ASE a interação temperatura e tempo de ativação (12) apresentou efeito significativo negativo, ou seja, a combinação de dois efeitos principais positivos gerou uma interação de dois fatores negativos, indicando que se as duas variáveis foram aumentadas simultaneamente haverá uma diminuição na ASE. O resultado da Análise de Variância para a resposta ASE evidencia a falta de ajuste dos dados a um modelo linear, apresentando como superfície de resposta uma superfície convexa. 


\section{REFERÊNCIAS BIBLIOGRÁFICAS}

AQUINO, D. F. Sisal - Proposta de Preço Mínimo 2012/2013: Conab, 2012 - Estudos Internos.

ASADULlAH, M.; ASADUZZAMAN, KABIR, M.S.; MOSTOFA, M.G.; MIYAZAWA, T. Chemical and structural evaluation of activated carbon prepared from jute sticks for Brilliant Green dye removal from aqueous solution. J. Hazard. Mater., v. 174, p. 437-443, 2010.

ARRUDA FILHO, N.; DANTAS, C.P.; LEAL, A.F.; BARBOSA, N.P.; SILVA, C.G.; ALEXANDRE, M.V. Resistência mecânica de compósitos cimentícios leves utilizando resíduos industriais e fibras de sisal. Revista Brasileira de Engenharia Agrícola e Ambiental, v. 16, n. 8, p. 894-902, 2012.

BARROS NETO, B.; SCARMINIO, J.B.; BRUNS, R.E. Como fazer experimentos: Pesquisa e desenvolvimento na ciência e na indústria. 2a Ed., Campinas: Editora Unicamp,. 2003.

CHEN, S.; ZENG, H. Improvement of reduction capacity of activated carbon fiber. Carbon, v. 41, p. 1265-1271, 2003.

FARIAS FILHO, J.; TOLEDO FILHO, R.D.; LIMA, P.R.L. Efeito da argila calcinada sobre a durabilidade de argamassas reforçadas com fibras curtas de sisal. Revista Brasileira de Engenharia Agrícola e Ambiental, v. 14, p. 1109-1114, 2010.

KU, H.; WANG, H.; PATTARACHAIYAKOOP, N.; TRADA, M. A review on the tensile properties of natural fiber reinforced polymer composites. Composites: Part B, v. 42, p. 856-873, 2011.

LI, Y.; MAI, Y.M.; YE, L. Sisal fibre and its composites a review of recent developments. Composities Sci. Technol., v. 60, p. 2037-2055, 2000.

MESTRE, A.S.; BEXIGA,A.S.; PROENÇA, M.; ANDRADE, M.; PINTO. M.L.; MATOS, I.M.F.; CARVALHO, A.P. Activated carbons from sisal waste by chemical activation with K2CO3: kinetics of paracetamol and ibuprofen removal from aqueous solution. Bioresour. Technol., v. 102, p. 8253-8260, 2011.

MOHAMED, A. R.; MOHAMMADIB, M.; DARZI, G. N. Preparation of carbon molecular sieve from lignocellulosic biomass. Renewable Sustainable Energy. Rev,, v.14, p. 1591-1599, 2010.

OASHI, M.C.G. Estudo da cadeia produtiva como subsídio para pesquisa e desenvolvimento do agronegócio do sisal na Paraíba. 1999. Tese (Doutorado em Engenharia de Produção) Universidade Federal de Santa Catarina, Florianópolis, 1999.

PHAN, N.H.; RIO, S.; FAUR, C.; LE COQ L.; LE CLOIREC; NGUYEN, T.H. Production of fibrous activated carbons from natural cellulose (jute, coconut) fibers for water treatment applications. Carbon, v. 44, p. 2569-2577, 2006

RAMESH, M.; PALANIKUMAR, K.; HEMACHANDRA REDDY, K. Mechanical property evaluation of sisal-jute-glass fiber reinforced polyester composites. Composites: Part B, v. 48, p. 1-9, 2013. 